\title{
Género, complementariedades y exclusiones en Mesoamérica y los Andes
}

doi: http://dx.doi.org/10.32870/

espiral.v22i62.259.g289

Natalia Castelnuovo Biraben•

Resultado de una serie de diálogos y puentes entre académicos del Norte y el Sur, activistas, e intelectuales de organizaciones y comunidades indígenas, Género, complementariedades $y$ exclusiones en Mesoamérica y los Andes es además de una importante contribución al campo de estudios sobre pueblos indígenas y género en América Latina, una invitación a reflexionar acerca de los nuevos desafíos que plantean escenarios caracterizados por una escalada de violencia e inseguridad, el avance de un modelo de desarrollo extractivista, y el de la reciente aparición pública, en diciembre pasado, del Ejército Zapatista de Liberación Nacional, entre otros. Al menos estos fueron algunos de los tópicos enfatizados por varias de sus autoras durante la presentación del libro organizada por los Seminarios de Género y Etnicidad, y Antropología Jurídica.

Con esa misma intención que tuvo esta obra colectiva de reunir a autores con diversos enfoques, metodologías, trayectorias y experiencias, se llevó adelante una presentación polifónica que no solo reflejó los diálogos entre académicos, activistas y miembros de los pueblos indígenas, sino

Aída Hernández y Andrew Canessa (2012). Género, complementariedades y exclusiones en Mesoamérica y los Andes. Perú: International Work Group for Indigenous Affairs (IWGIA) / Abya Yala Press / The British Academy.

Espiral, Estudios sobre Estado y Sociedad

Vol. xxII No. 62 • Enero / Abril de 2015 
que además dejó entrever los distintos posicionamientos que mujeres indígenas están adoptando en sus localidades para enfrentar los problemas que afectan cotidianamente sus comunidades.

Ese abanico de respuestas que las mujeres indígenas desde sus respectivos hogares, organizaciones de base, pueblos y espacios de participación local, regional y nacional, están dando ante los embates de multinacionales, privados y Estados-nación que incluso 're-conociendo' sus derechos actúan como si los desconocieran, puso una vez más en evidencia su creatividad transformadora e innegable capacidad de resistencia. ¿De qué otra forma si no explicando la infinidad de estrategias, propuestas alternativas y procesos de lucha que son impulsados y protagonizados por mujeres indígenas? Tal como señaló Rufina Villa Hernández, una de las coautoras además de presentadora de la obra, haciendo referencia a cómo se encuentran las mujeres de la sierra de Cuetzalan, Puebla, frente a los avances de proyectos mineros e hidroeléctricos que amenazan a sus pueblos:

Como organización seguimos trabajando por los derechos colectivos de nuestras comunidades y vemos que la fuerza de las mujeres está ahí. Las mujeres de la comunidad están participando en la resistencia por la defensa de su territorio, en la defensa de un río en donde quieren construir hidroeléctricas. $Y$ ellas están a la par con los hombres, apoyando para que no pase la máquina que quiere abrir camino para empezar a construir la hidroeléctrica. Esto se está dando en varios lugares de la sierra. $Y$ como el tema de la justicia y de los derechos de nuestros pueblos, creemos que es importante seguir en esta lucha, seguir apoyando, defendiendo para que podamos sacar adelante los proyectos que a nosotros sí nos interesan.

La proliferación de respuestas que, desde diferentes ámbitos y con diverso grado de injerencia, vienen dando las mujeres indígenas puede entenderse como expresión de un proceso 
de participación política en un sentido más amplio que tiene como horizonte la autonomía, autodeterminación y justicia comunitaria, como asimismo el reconocimiento de derechos de las mujeres: el de la herencia, el acceso a la tierra, la participación política y una vida libre de violencia doméstica. Pero si bien es posible identificar la adopción de discursos y prácticas que reconocen el valor y sus derechos, esto no significa que en el camino las mujeres no hayan tenido que enfrentar un repertorio de obstáculos y tensiones vinculados a nociones propias y ajenas de 'cultura' y/o a los papeles tradicionalmente asignados a hombres y mujeres, por tan solo nombrar algunos de ellos. A estas dificultades que las mujeres han ido superando se refirió Liliana Vianey Vargas durante la presentación cuando describió la discriminación de género hacia las mujeres mixe en el modus operandi de los impartidores de justicia en el municipio de Tlahuitoltepec: "Había ese sesgo de género que al final de cuenta se les decía a las mujeres que reflexionaran y que regresaran a sus casas. Era una constante. Pero lo cierto es que poco a poquito las mujeres han ido haciendo usos de sus derechos, de estos discursos de derechos de las mujeres[...]".

La experiencia de las mujeres mixe presenta algunas similitudes con la de las nahuas, en tanto que paralelamente a la formación en sus derechos específicos como mujeres ellas han ido abriéndose un camino en la construcción de un proyecto propio para lidiar con los problemas y necesidades de sus pueblos y aquellos que las afectan específicamente como mujeres. En esas reuniones entre "compañeras y amigas del pueblo y de la región -contó Amalia Romay, al mismo tiempo que destacó el papel pionero que desempeñó su madre en el proceso organizativo de Defensa Popular de Oteapan- las mujeres encontraron un espacio colectivo de reflexión acerca de su vida cotidiana y de elaboración de una visión de oportunidades colectivas". Los logros que ellas alcanzaron pusieron de relieve el potencial que su 
organización les ofrecía para hacer escuchar sus voces. En palabras de Amalia Mendoza Romay, "la mujer siempre tiene un papel de representación silenciosa, pero siempre ha estado y generado los cambios más importantes de los pueblos".

El libro y los talleres que lo precedieron tuvieron como uno de sus objetivos la posibilidad de debatir en torno a dos enfoques contrapuestos que han permeado los estudios sobre género en pueblos indígenas: el de la complementariedad en la región andina boliviana y peruana, y el de los derechos de las mujeres en Mesoamérica. Así, mientras que las culturas andinas ofrecían una fuerte resistencia al discurso de los derechos, la discusión acerca de la desigualdad y la posibilidad de construir relaciones equitativas impulsada fundamentalmente desde el levantamiento zapatista encontró eco entre mujeres mexicanas y guatemaltecas. Un reflejo de estas reflexiones y diversidad de miradas es la que encontramos en el libro. En el primer capítulo Pamella Calla nos introduce en la experiencia de la Asamblea Constituyente en Bolivia y del papel desempeñado por las mujeres en este proceso de construcción de ciudadanía. Siguiendo los estudios en Bolivia, Patricia Chávez León et al. nos invitan a pensar acerca de cuáles son los desafíos que enfrentan las mujeres bolivianas para construir una agenda feminista autónoma. Para la amazonia boliviana contamos con el artículo de Esther López, quien analiza la manera en la que las mujeres tacanas construyen sus liderazgos al mismo tiempo que enfrentan las desigualdades de género. A este capítulo le sigue el de Andrew Canessa abordando desde una perspectiva de género el pensamiento político de un intelectual aymara. Finalmente el bloque de estudios andinos se cierra con el trabajo sobre Perú de Patricia Oliart, con un análisis sobre el significado y apropiación que las mujeres indígenas han hecho de los discursos globales de derechos en función de sus propias luchas. El siguiente 
Género, complementariedades y exclusiones en Mesoamérica y los Andes

bloque de la región Mesoamericana abre con un texto de Aura Cumen donde se exploran las contradicciones de la justicia comunitaria y las presentaciones racistas que de la misma se han hecho en la prensa guatemalteca, tomando como ejemplo las experiencias de mujeres k’iche. Rachel Sieder y Morna Macleod son las autoras del próximo artículo centrado en analizar la importancia de la cosmovisión maya en la reconstitución del derecho indígena y de la justicia comunitaria en Guatemala. El último trabajo de esta sección es el de Rolando Chacach, con una propuesta que indaga en los encuentros y desencuentros entre mayanismo y feminismo.

Los próximos seis capítulos se abocan al estudio de experiencias indígenas mesoamericanas en México. María Teresa Sierra, Felicitas Martínez y Paula Florentino, en sus respectivos trabajos, nos introducen en la experiencia semiautónoma de justicia comunitaria en Guerrero. Por su parte, Rufina Villa, presenta la historia de su organización y relata entre los logros alcanzados por las mujeres organizadas la influencia en el Juzgado Indígena. En el siguiente capítulo Martha Sánchez narra su propia experiencia como dirigente nacional de la Asamblea Nacional Indígena Plural por la Autonomía. El libro continúa con los estudios de Ángela Ixkic Duarte y Amalia Romay, quienes focalizan en los logros de las mujeres nahuas para incluir sus propias agendas en el marco de procesos organizativos más amplios de sus pueblos. Liliana Vianey Vargas y Carolina María Vásquez contribuyen con sus respectivos textos acerca de los usos de la cultura indígena como herramienta de lucha y retos que encuentran las mujeres en la demanda de sus propios espacios de justicia comunitaria. A modo de cierre, Alejandro Cerda presenta en su texto las experiencias de las mujeres zapatistas en las regiones autónomas y la importancia que tuvo el movimiento zapatista para colocar en la agenda del movimiento indígena mexicano las demandas 
específicas de las mujeres. Como dicen las compañeras en Chiapas, refiriéndose a la lucha que han dado porque los derechos colectivos incluyan sus necesidades especificas como mujeres: que tengan "ojos y corazón de mujer". 